\title{
Toward a Fully Lagrangian Atmospheric Modeling System
}

\author{
Jahrul M. Alam AND John C. Lin \\ Department of Earth and Environmental Sciences, University of Waterloo, Waterloo, Ontario, Canada
}

(Manuscript received 27 December 2007, in final form 17 March 2008)

\begin{abstract}
An improved treatment of advection is essential for atmospheric transport and chemistry models. Eulerian treatments are generally plagued with instabilities, unrealistic negative constituent values, diffusion, and dispersion errors. A higher-order Eulerian model improves one error at significant cost but magnifies another error. The cost of semi-Lagrangian models is too high for many applications. Furthermore, traditional trajectory "Lagrangian" models do not solve both the dynamical and tracer equations simultaneously in the Lagrangian frame. A fully Lagrangian numerical model is, therefore, presented for calculating atmospheric flows. The model employs a Lagrangian mesh of particles to approximate the nonlinear advection processes for all dependent variables simultaneously. Verification results for simulating seabreeze circulations in a dry atmosphere are presented. Comparison with Defant's analytical solution for the sea-breeze system enabled quantitative assessment of the model's convergence and stability. An average of 20 particles in each cell of an $11 \times 20$ staggered grid system are required to predict the two-dimensional sea-breeze circulation, which accounts for a total of about 4400 particles in the Lagrangian mesh. Comparison with Eulerian and semi-Lagrangian models shows that the proposed fully Lagrangian model is more accurate for the sea-breeze circulation problem. Furthermore, the Lagrangian model is about 20 times as fast as the semi-Lagrangian model and about 2 times as fast as the Eulerian model. These results point toward the value of constructing an atmospheric model based on the fully Lagrangian approach.
\end{abstract}

\section{Introduction}

A quantitative understanding of physical and chemical processes in the atmosphere is essential in order to assess the human impact on the environment. For example, the accumulation of greenhouse gases (GHG) (e.g., $\mathrm{CO}_{2}$ ) causes climate change by altering the earth's surface radiative balance, which in turn can change the global and regional patterns of sources and sinks of GHGs (Denning et al. 1996). Large-scale transport and chemistry models are essential tools in investigating various challenging issues in atmospheric science. To accurately simulate the concentration of trace gases and to determine their sources/sinks, it is necessary to improve current atmospheric models used to simulate the transport and chemical transformation of trace gases (Lin et al. 2003). Such models require numerical simu-

Corresponding author address: Jahrul Alam, Dept. of Earth and Environmental Sciences, University of Waterloo, 200 University Ave. W, Waterloo ON N2L3G1, Canada.

E-mail: alamj@uwaterloo.ca lation of advection-dominated flow problems. The realization that numerical treatment of advection on a conventional Eulerian mesh is plagued with instabilities and unrealistic negative constituent values has inspired continuous efforts in finding more elegant tools for improving atmospheric transport and chemistry models (Rood 1987; Wang and Hutter 2001).

The growing interest in computer modeling of the atmosphere for environmental problems underscores the importance of simulating advection adequately [e.g., see Ritchie (1986) for a discussion on a similar topic that arises in numerical weather prediction (NWP) models]. A better numerical treatment of the nonlinear advection process in atmospheric models is of central importance and an active area of research (Rood 1987; Garner 1989; Read et al. 2000; Jaouen 2007). Numerical time integration schemes adopted by atmospheric models can be classified into the following three categories: (i) Eulerian (e.g., chaper 4 in Chung 2002), (ii) semi-Lagrangian (e.g., chapter 7 in Behrens 2006), and (iii) fully Lagrangian (Garner 1989). Eulerian schemes-the use of finite-difference numerical 
methods on Eulerian meshes-compose a widely adopted approach in modeling advection (chapters 6 and 7 in Jacobson 1999). However, modeling advection on an Eulerian mesh leads to nonlinear instabilities due to spurious accumulation of energy at high wavenumbers (e.g., Phillips 1956; Hack 1992). A number of techniques have been proposed to stabilize Eulerian finitedifference schemes for advection (e.g., chapter 4 in Tannehill et al. 1997), but which amounted to severe numerical damping (e.g., Table 10.1 of Pielke 2002). For conditionally stable explicit Eulerian schemes, the maximum permissible time step is governed by stability consideration rather than the truncation error, thereby requiring many more time steps than would otherwise be the case (Staniforth and Cote 1991). In contrast, Lagrangian schemes (semi or fully Lagrangian) have, among others, the following advantages over naive Eulerian schemes: (i) The unconditional stability of these schemes permits adjusting a time step according to the need of having the temporal truncation error proportional to the spatial truncation error (Ritchie 1986; Bartello and Thomas 1996). (ii) Numerical diffusion is minimized without additional antidiffusion calculation, and gradients in tracer concentrations are preserved (Smolarkiewicz and Pudykiewicz 1992). Artificial damping or dispersion errors do not accumulate drastically because of the evaluation of advective terms (Rood 1987). (iii) Nonlinear instabilities can be dynamically removed by advecting parcels of fluid along the characteristic path lines (Alam 2000).

Semi-Lagrangian schemes were originally introduced to improve time-stepping criterion based on the accuracy requirement for low-resolution simulations of large-scale advection-dominated flow problems with quasigeostrophic dynamics (Wiin-Nielsen 1959; Sawyer 1963). These schemes are used in various disciplines with different names: "upstream interpolation method" (Mathur 1983; Mahrer and Pielke 1978), "trajectory method" (Krishnamurti 1962), "Eulerian-Lagrangian method" (Baptista 1987), "Lagrange-Galerkin method" (Bermejo 1990), or "characteristic Galerkin method" (Oliveira and Baptista 1995). Despite the improvement in time stepping, the integration of Lagrangian trajectories and the interpolation of advected fields introduce a large computational cost per time step, which increases drastically in 3D. The tradeoff between the computational overhead and improvement in accuracy for semi-Lagrangian simulations was studied extensively (e.g., see Pudykiewicz and Staniforth 1984; Bates and McDonald 1985; Staniforth and Pudykiewicz 1985; Ritchie 1986). For example, Bartello and Thomas (1996) reported that semi-Lagrangian schemes are about 5-10 times as costly relative to a classic second-order leapfrog scheme and verified that in the atmosphere, these schemes are inefficient at spatial scales below $300-400 \mathrm{~km}$ because of their considerable computational cost (Bartello and Thomas 1996). The interpolation in semi-Lagrangian schemes introduces computational damping that is equivalent to what would result from an Eulerian scheme: for example, linear interpolation on uniform mesh is equivalent to a first-order upwind scheme that damps out the numerical solution at large time (e.g., Crowley 1968; Bartello and Thomas 1996). One of the key objectives of the present study is to develop a cost-effective and accurate fully Lagrangian scheme.

The fully Lagrangian numerical methods-in which particles are tagged with physical properties of the fluid dynamical system - are often called "gridless" particle methods, making up a powerful class of computational fluid dynamics (CFD) techniques for multiscale flow simulations (e.g., Koumoutsakos 2005). These methods adopt a discretization of partial differential operators on a Lagrangian mesh of particles, thereby simulating the time evolution of advection terms in a fully Lagrangian framework such that no interpolation of advected fields is required. Particle methods construct a class of techniques for the numerical simulation of twoor three-dimensional, unsteady vortical flows of an incompressible fluid at high Reynolds number (Leonard 1985; Subramaniam 1996). Particle methods can also be conjoined with a grid without detracting from the fully Lagrangian character, but providing consistent, efficient, and accurate simulations (Koumoutsakos 2005).

A fully Lagrangian particle method would be an alternative to semi-Lagrangian or Eulerian simulations. While Lagrangian methods have been considered for oceanic modeling (e.g., see Dritschel et al. 1999; Haertel and Randall 2002; Haertel et al. 2004), little has been done to develop and verify a suitable particle-based method in an atmospheric setting. Previous attempts at Lagrangian, particle-based atmospheric models found applications in dispersion modeling, using air parcels of unit mass to determine the evolution of a concentration field (Sawford 1985; Wilson and Sawford 1995). These models rely on a pregenerated wind field that is either interpolated from the observed data or from a separate Eulerian model's output (e.g., Lin et al. 2003; Fast and Easter 2006; Stohl and Thomson 1999). Therefore, the entire atmospheric dynamical system is not simulated in the Lagrangian frame. For instance, Lange (1978) presented a three-dimensional particle-in-cell model in which the concentration field was advected by a wind field supplied by an accompanying non-Lagrangian code called the mass-adjusted three-dimensional wind field (MATHEW; Sherman 1978). Further examples in- 
clude such commonly used models as the Stochastic Time Inverted Lagrangian Transport model (STILT; Lin et al. 2003), "FLEXPART" (Stohl et al. 1998), the Hybrid Particle and Concentration Transport Model (HYPACT; Uliasz 1996), Hybrid Single Particle Lagrangian Integrated Trajectory model (HYSPLIT; Draxler and Hess 1998), and Numerical Atmospheric Dispersion Modeling Environment (NAME; Ryall and Maryon 1998).

Despite the aforementioned considerable advantages of Lagrangian particle methods, to our knowledge no atmospheric model simulating mesoscale phenomena exists that is based on the "fully Lagrangian" formulation. In this paper we present a fully Lagrangian model for simulating atmospheric motion. First, the present work uses a fully Lagrangian scheme for the advective time evolution of the flow using a Lagrangian mesh of particles. Second, a simultaneous solution of all state variables (e.g., velocity and scalar field) is carried out in the Lagrangian framework, which is a clear step beyond conventional trajectory models (e.g., Lin et al. 2003) that do not solve the equations of motion.

In section 2 we present a detailed description of the proposed fully Lagrangian modeling system. Our approach is based on splitting the nonlinear advection process from all other properties of motion. The developed scientific contribution needs to be validated and verified, which is presented in section 3 . In addition, we have compared the performance of the developed fully Lagrangian model with respect to two reference models-one is Eulerian and the other is semi-Lagrangian (section 4). Finally, we conclude this paper by summarizing our observations and findings in section 5 , where we discuss some potential future research directions guided by the present study.

\section{Model description}

\section{a. Equations of motion-General form}

At the heart of an atmospheric model, a set of conservation principles form a coupled set of partial differential equations (PDEs) that must be solved simultaneously. A system of prognostic equations describing atmospheric motion can be compactly written as

$$
\frac{\partial \boldsymbol{\Psi}}{\partial t}+\mathbf{u} \cdot \boldsymbol{\nabla} \boldsymbol{\Psi}=\mathbf{R}
$$

where $\boldsymbol{\Psi}$ is a vector of $d$ state variables, $\mathbf{u}$ is a two- or three-dimensional velocity vector, and $\mathbf{R}$ is a vector that represents all forces or sources (e.g., diffusion and chemical reaction). Typically, the components of $\boldsymbol{\Psi}$ are velocity, density, potential temperature, and/or trace gas concentration (e.g., see chapter 2 in Pielke 2002).
In a Lagrangian framework, Eq. (1) can be written as

$$
\frac{d \boldsymbol{\Psi}}{d t}=\mathbf{R},
$$

where $(d / d t)$ is known as the material derivative-the derivative following fluid parcel motion along trajectories (e.g., chapter 3 in Kundu 1990). In the absence of external forces or source/sink terms: $\mathbf{R}=0$, which implies that

$$
\frac{d \Psi}{d t}=0
$$

Equation (3) states that the quantity $\boldsymbol{\Psi}$ is invariant along trajectories of a flow, building the foundation for Lagrangian numerical modeling.

In the present fully Lagrangian model, we approximate the motion according to Eq. (2) in two stages during each time step. In the first stage of the time step we assume that an atmospheric state is governed by inertial forces only [e.g., Eq. (3)]. Therefore, an air parcel in motion moves with the current velocity without changing $\boldsymbol{\Psi}$ within the parcel. In the second stage of the time step, we neglect the motion of air parcels and consider that an atmospheric state is at rest, where flow properties defined by $\boldsymbol{\Psi}$ changes within a parcel according to external sources/sinks or forces represented by nonzero $\mathbf{R}$. The implementation is described in detail in sections $2 b$, c.

\section{b. Stage I-Modeling of advection by parcel movement}

To satisfy Eq. (3), a finite collection of air parcelsobjects carrying the physical property $\boldsymbol{\Psi}$ of an atmospheric system - represented by a Lagrangian mesh of particles are used to model the flow in a computational region. The mathematical details for discretizing dynamical equations on a mesh of Lagrangian particles is given by Koumoutsakos (2005). Examples of such techniques are vortex methods (VMs; Cottet and Koumoutsakos 2000) and smooth particle hydrodynamics (SPHs; Lucy 1977; Monaghan 1988). The calculation at stage I is adopted from such CFD techniques.

\section{1) Setup of Air PARCEl CONFIGURATION}

Let us denote the position of $\mathcal{N}$ air parcels at time $t$ by $x_{i}(t)$, where $i=1, \ldots, \mathcal{N}$. Each parcel moves along an individual trajectory with the tagged velocity, thereby constructing a Lagrangian mesh of particles. None of the tagged quantities is changed during the movements of parcels because all other effects are neglected (i.e., $\mathbf{R}=0$ ). The parcel arrangement at $t=t_{0}$ and the displacement, at $t=t_{0}+\Delta t$, are shown in Fig. 1 schematically, where parcels whose positions at $t=t_{0}$ 


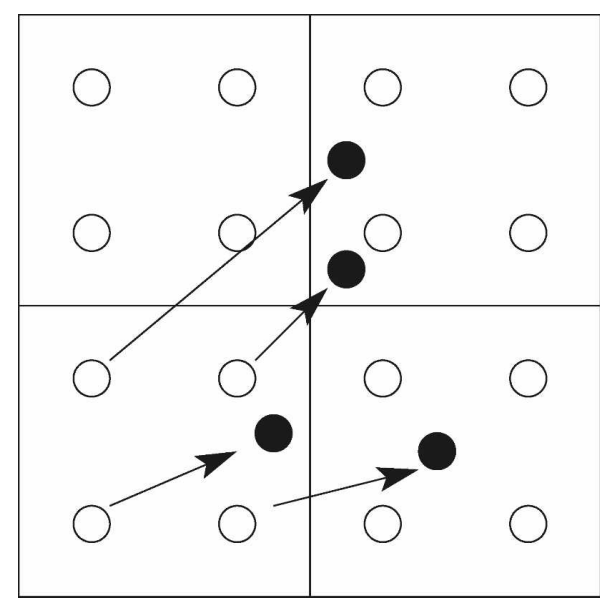

FIG. 1. Movement of parcels in a typical two-dimensional model is presented schematically. A nonfilled circle represents a parcel assigned at $t=t_{0}$. A filled circle represents a parcel at $t=t_{0}+\Delta t$. Arrows link present position with future positions. For simplicity, the movement of only four parcels is presented. Each parcel is tagged with velocity, temperature, tracer concentration, etc., as needed for a particular flow.

(nonfilled circles) are linked to positions at $t=t_{0}+\Delta t$ (filled circles) by arrows. Movement of only four parcels are illustrated. In practice, parcels are also arranged nonuniformly at $t=t_{0}$ following the initial density profile of the atmosphere.

During this stage, the advective flow evolution within a time step $\Delta t$ is determined by rearranging the Lagrangian mesh of particles according to the new position of parcels.

\section{2) PARCEL AdVECTION}

Let $\mathbf{u}_{i}\left[\mathbf{x}_{i}\left(t_{0}\right)\right]$ be the velocity of a parcel with position $\mathbf{x}_{i}\left(t_{0}\right)$ at time $t_{0}$. The trajectory of $i$ th parcel satisfies the following ordinary differential equation (ODE):

$$
\frac{d \mathbf{x}_{i}}{d t}=\mathbf{u}_{i}\left(\mathbf{x}_{i}, t_{0}\right)
$$

New positions $\mathbf{x}_{i}\left(t_{0}+\Delta t\right)$ of parcels for $i=1, \ldots, \mathcal{N}$ after a certain time step $\Delta t$ are given by an explicit finite-difference integration of Eq. (4):

$$
\mathbf{x}_{i}\left(t_{0}+\Delta t\right)=\mathbf{x}_{i}\left(t_{0}\right)+\mathbf{u}_{i}\left[\mathbf{x}_{i}\left(t_{0}\right)\right] \Delta t,
$$

where $\mathbf{u}_{i} \Delta t$ is the displacement over the time step $\Delta t$.

According to Eq. (3), the advection of air parcels preserves a tagged property $\boldsymbol{\Psi}_{i}$ of the $i$ th parcel along the parcel trajectory. So at the new parcel location $\mathbf{x}_{i}\left(t_{0}+\Delta t\right)$, we must have

$$
\boldsymbol{\Psi}\left[\mathbf{x}_{i}\left(t_{0}+\Delta t\right)\right]_{i}=\boldsymbol{\Psi}_{i}\left[\mathbf{x}_{i}\left(t_{0}\right)\right],
$$

which implies that the parcel located at $\mathbf{x}_{i}\left(t_{0}\right)$ is moved to the position $\mathbf{x}_{i}\left(t_{0}+\Delta t\right)$. Note that the quantity $\boldsymbol{\Psi}_{i}$ does not change because of the numerical treatment of advection. The calculation associated with advection is dictated by the physics of the flow. The error in the Lagrangian treatment of advection is linked with the error in obtaining Eq. (5) (i.e., how accurately the new parcel position is determined).

Notable features to distinguish the present formulation from currently available Lagrangian atmospheric or numerical weather prediction models are following. (i) An individual parcel is advected along an approximate trajectory [Eq. (5)], avoiding steep computational cost of semi-Lagrangian interpolation (Ritchie 1986). In other words, the advection is approximated on a fully Lagrangian mesh of particles. (ii) Equation (6) updates all state variables at the new parcel position, ensuring a simultaneous treatment of advection in the Lagrangian mesh. Therefore, this approach ensures simulations of the atmosphere in a fully Lagrangian manner-a need of currently available Lagrangian models for tracer transport problems (Lin et al. 2003).

The fully Lagrangian treatment of advection presented in this section is accompanied by a second stage of the time step, during which all effects neglected during the first stage are modeled.

\section{c. Stage II-Time evolution of state variables based on stationary air parcels}

The Lagrangian mesh of particles advected in stage I is now a set of Eulerian collocation points because particles are not moved at stage II of time step. The time evolution of the flow is determined by solving Eq. (2) (without the advective terms) on such mesh (e.g., see Koumoutsakos 2005). However, additional care must be taken to approximate differential operators (e.g., diffusion or gradient terms) contained in $\mathbf{R}$ on such nonuniform set of Eulerian collocation points. We adopt an Eulerian staggered finite-difference grid system (e.g., Harlow and Welch 1965), which is presented in Fig. 2. It is thus necessary to link between the particle mesh and the staggered grid. As a straightforward approach, an approximate solution tagged with particles in a cell is averaged to find an approximate solution at the centroid of this cell, which is used for finitedifference approximation of $\mathbf{R}$ on the staggered grid. Linear effects are also assumed to be uniform for all parcels within an individual cell. This is depicted in Fig. 2 , where "aligned dots" are used to indicate the cell around a grid point.

When the incompressibility of the flow is incorporated in the system in Eq. (2), the prognostic mass- 


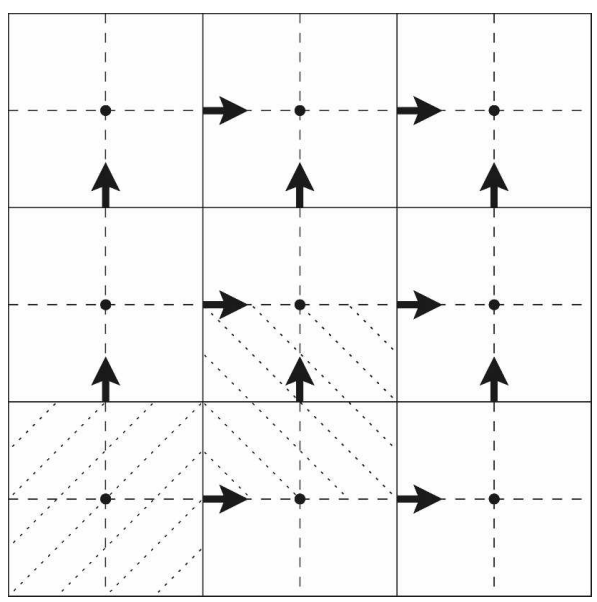

FIG. 2. The staggered grid system, where horizontal and vertical velocities are calculated at grid points marked by arrows, and scalar quantities such as pressure, potential temperature, etc., are calculated at grid points marked by a "dot." A cell around a dot is shown by right-aligned dotted lines, and that for an "upward arrow" is shown by left-aligned dotted lines. A quantity is calculated at a grid point by averaging quantities tagged with parcels in the cell around this grid point as shown by aligned dots for two individual cells.

conservation equation reduces to a diagnostic form and one must consider a scheme such that the mass conservation is satisfied. There are three widely used approaches: (i) treatment of velocity and pressure on a uniform staggered finite-difference grid system (Harlow 1964), (ii) using a projection scheme for velocity correction (Shen 1992), or (iii) transforming the model equations in terms of vorticity, removing the pressure gradient term (e.g., chapter 9 in Tannehill et al. 1997). In the present work, we consider the velocity-pressure formulation to describe mathematically the motion of the atmosphere. Harlow and Welch (1965) showed that the staggered grid system in Fig. 2 ensures the mass conservation in a two-dimensional incompressible flow simulation. Note that the grid does not detract from the character of the Lagrangian particle-based method, but enhances consistent, efficient, and accurate simulations (Koumoutsakos 2005).

\section{TIME EVOLUTION OF STATE VARIABLES}

The time evolution of state variables can be calculated using a standard time marching scheme. Using an explicit Euler's method, Eq. (2) becomes

$$
\boldsymbol{\Psi}_{i}^{n+1}=\boldsymbol{\Psi}_{i}^{n}+\Delta t \mathbf{R}_{i},
$$

where a superscript " $n$ " represent a discrete time level and $i$ denotes the parcel position. Note that the scheme in (7) is conditionally stable, which may be relaxed us- ing a fully implicit scheme. One sees that the time evolution at stage II is performed on the Eulerian mesh of particles, retaining the character of a particle-based method.

\section{Simulations and verification}

\section{a. Sea-breeze model: Governing equations}

The system of atmospheric equations used to test and verify the Lagrangian model is based on a sea-breeze model by Defant (1951), but including consideration of nonlinear advection terms (Martin and Pielke 1983). This model was derived by neglecting moisture.

The governing equations represent a twodimensional motion in coastal regions in terms of mesoscale perturbations from the synoptic state of all dependent variables, where the synoptic state is indicated by the subscript zero. The decomposition $\Psi=\Psi_{0}+$ $\Psi^{\prime}+\Psi^{\prime \prime}$ contains a subgrid-scale perturbation $\Psi^{\prime \prime}$ in addition to the mesoscale perturbation $\Psi^{\prime}$. However, all terms containing subgrid-scale perturbations (e.g., subgrid-scale momentum and heat fluxes) are parameterized in terms of mesoscale perturbation quantities (e.g., chapter 5 in Pielke 2002). Therefore, we have dropped "primes" in the following description. We now write down the system of equations in component form [rather than the more general form of Eq. (1)], where all the dependent variables representing mesoscale perturbations are defined in Table 1:

$$
\begin{aligned}
\frac{\partial u}{\partial t}+\left[u \frac{\partial u}{\partial x}+w \frac{\partial w}{\partial z}\right] & =-\alpha_{0} \frac{\partial p}{\partial x}+f v-\sigma_{x} u, \\
\frac{\partial w}{\partial t}+\left[u \frac{\partial w}{\partial x}+w \frac{\partial w}{\partial z}\right] & =\gamma \theta-\alpha_{0} \frac{\partial p}{\partial z}-\sigma_{z} w \\
\frac{\partial u}{\partial x}+\frac{\partial w}{\partial z} & =0 \\
\frac{\partial v}{\partial t}+\left[u \frac{\partial v}{\partial x}+w \frac{\partial w}{\partial z}\right] & =-f u-\sigma_{y} v, \quad \text { and } \\
\frac{\partial \theta}{\partial t}+\left[u \frac{\partial \theta}{\partial x}+w \frac{\partial \theta}{\partial z}\right]+\beta w & =\kappa\left\{\frac{\partial^{2} \theta}{\partial x^{2}}+\frac{\partial^{2} \theta}{\partial z^{2}}\right\} .
\end{aligned}
$$

Periodic boundary conditions are assumed for all dependent variables in the $x$ direction. The velocity components satisfy a no-slip condition at $z=0$. The model region is truncated at $z=z_{\max }$, where a rigid-lid condition is assumed. For all numerical experiments, we prescribe a potential temperature:

$$
\theta(x, z, t)=\left\{\begin{array}{cc}
M e^{i \omega t} \sin \left(k_{x} x\right) & \text { if } z=0 \\
0 & \text { otherwise }
\end{array}\right.
$$


TABLE 1. Definition for all variables and constants for the model equations

\begin{tabular}{|c|c|c|}
\hline \multicolumn{3}{|c|}{ Independent variables } \\
\hline$x$ & $(\mathrm{~m})$ & Horizontal coordinate \\
\hline$z$ & $(\mathrm{~m})$ & Vertical coordinate \\
\hline$t$ & (s) & Time \\
\hline \multicolumn{3}{|c|}{ Dependent variables } \\
\hline$u$ & $\left(\mathrm{~m} \mathrm{~s}^{-1}\right)$ & $x$ component of velocity \\
\hline$v$ & $\left(\mathrm{~m} \mathrm{~s}^{-1}\right)$ & $y$ component of velocity \\
\hline$w$ & $\left(\mathrm{~m} \mathrm{~s}^{-1}\right)$ & $z$ component of velocity \\
\hline$p$ & $(\mathrm{hPa})$ & Pressure field \\
\hline$\theta$ & (K) & Potential temperature \\
\hline \multicolumn{3}{|l|}{ Constants } \\
\hline$g$ & $\left(\mathrm{~m} \mathrm{~s}^{-2}\right)$ & Acceleration due to gravity \\
\hline$\theta_{0}$ & $(\mathrm{~K})$ & $\begin{array}{l}\text { Synoptic-scale potential } \\
\text { temperature }\end{array}$ \\
\hline$\beta$ & $\left(\mathrm{K} \mathrm{m}^{-1}\right)$ & $\begin{array}{l}\text { Vertical gradient of } \\
\text { synoptic-scale potential } \\
\text { temperature }\end{array}$ \\
\hline$\gamma$ & $\left(\mathrm{m} \mathrm{K} \mathrm{s}^{-2}\right)$ & $g / \theta_{0}$ \\
\hline$\alpha_{0}$ & $\left(1 \mathrm{~m}^{-3}\right)$ & $\begin{array}{l}\text { Synoptic-scale reference } \\
\text { specific volume }\end{array}$ \\
\hline$M$ & $\left({ }^{\circ} \mathrm{C}\right)$ & $\begin{array}{l}\text { Max temperature } \\
\text { difference between land } \\
\text { and ocean }\end{array}$ \\
\hline$k_{x}$ & $\left(1 \mathrm{~m}^{-1}\right)$ & Horizontal wavenumber \\
\hline$\omega$ & $\left(1 \mathrm{~s}^{-1}\right)$ & $\begin{array}{l}\text { Frequency for temporal } \\
\text { periodic variation }\end{array}$ \\
\hline$\sigma_{x}, \sigma_{y}, \sigma_{z}$ & $\left(1 \mathrm{~s}^{-1}\right)$ & $\begin{array}{l}\text { Coef to parameterize } \\
\text { subgrid-scale flux terms }\end{array}$ \\
\hline
\end{tabular}

(see Table 1 for the definition of symbols). A number of numerical experiments are performed using this seabreeze system for validation and verification of the proposed fully Lagrangian modeling approach.

\section{b. Comparison with Defant's analytical, linear model}

A critical test of the Lagrangian model was carried out by examining whether the proposed model predicts similar results as compared with analytical solutions obtained by Defant's linear theory. A linear model that is similar to the one presented in Defant (1951) can be obtained from the governing Eqs. (8)-(12) by removing all nonlinear terms indicated in brackets. Analytical solutions of the Defant's model are obtained in Martin and Pielke (1983) by assuming periodic boundary conditions both in the horizontal direction and with respect to a diurnal time span. In the vertical direction, a vanishing solution was assumed.

To set up such an experiment, all parcels were kept stationary at their initial locations, which is equivalent to bypassing the advection computation in stage I (section $2 \mathrm{~b}$ ). Therefore, quantities tagged with each air parcel are evolved in time (excluding advection terms) dur- ing the calculation in stage II. To be consistent with the analytical solutions, a periodic boundary condition was chosen for the horizontal direction covering a distance of $100 \mathrm{~km}$ over land and sea, which was accompanied by a no-slip condition for the vertical velocity $w$ at ground. In the vertical direction, the model top was set at a fixed height (e.g., $4 \mathrm{~km}$ for this experiment), where a rigid-lid approximation was applied. Note that the rigid-lid approximation is inconsistent with the original Defant's analytical model.

The experiment predicts five dependent variables: horizontal components of the wind fields $u(x, z, t)$; and $v(x, z, t)$; vertical wind field $w(x, z, t)$; pressure $p(x, z, t)$; and the potential temperature $\theta(x, z, t)$, where all variables are assumed to be independent of the $y$ direction. Necessary parameters that are used to initialize the model can be found in chapter 5 in Pielke (2002). In Fig. 3, we present the potential temperature and horizontal velocity fields calculated in the above experiment at $t=6 \mathrm{~h}$ ( $6 \mathrm{~h}$ after sunrise). For the purpose of verification, we have reproduced equivalent results for the Defant model using analytical solutions of the system. Despite excellent correspondence between numerical and analytical results, we stress here that inherent differences exist between the analytical solutions and the Lagrangian numerical model. In deriving Defant's analytical model, a solution vanishing with altitude was assumed for mesoscale perturbations (e.g., for the vertical velocity and the potential temperature):

$$
w(x, z, t) \rightarrow 0, \text { as } z \rightarrow \infty,
$$

and

$$
\theta(x, z, t) \rightarrow 0, \text { as } z \rightarrow \infty
$$

for all $x$ and $t$. In contrast, the numerical model adopted a rigid-lid boundary condition because of its finite vertical extent. Therefore, disagreement between the analytical solutions and the Lagrangian numerical model near the model top $\left(z=z_{\max }\right)$ is expected because of differences in the boundary conditions. Such an error can also be synchronized to the entire model domain. This numerical experiment, however, confirms that the present model predicts the dynamics of coastal circulation, which are consistent with those predicted by the linear theory of Defant (1951). Note that present simulations adopt a particle mesh that was fixed in time. These results are within good qualitative agreement with simulations presented in Fig. 5 of Martin and Pielke (1983), where an Eulerian uniform finitedifference mesh was used.

Let us now discuss the model's performance, when the movement of air parcels is retained during the stage I calculation. 
(a)

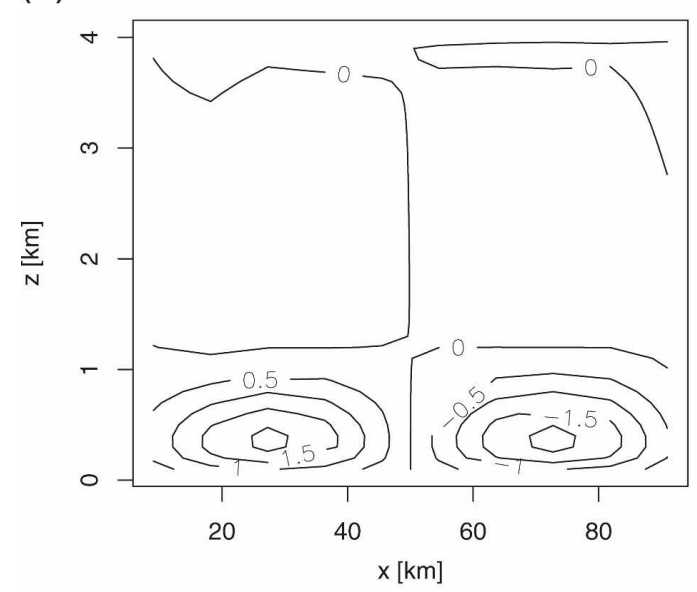

theta(analytical); $\mathrm{t}=6[\mathrm{~h}]$;

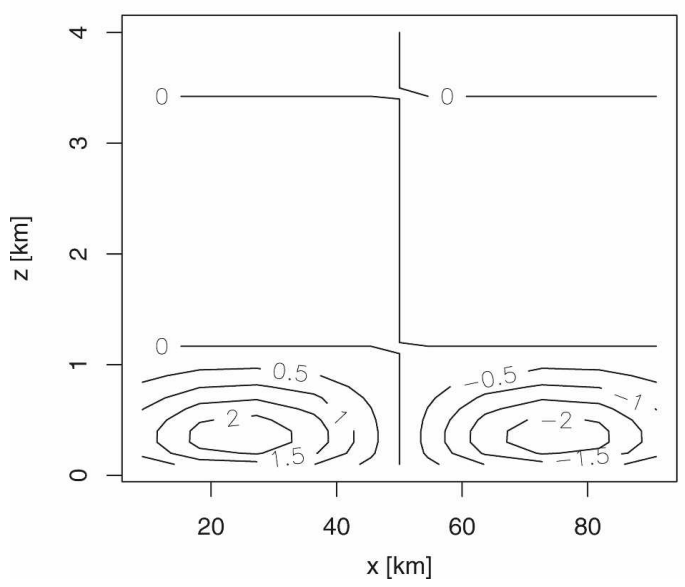

(b)

$\mathrm{u}$ (numerical); $\mathrm{t}=6[\mathrm{~h}]$;

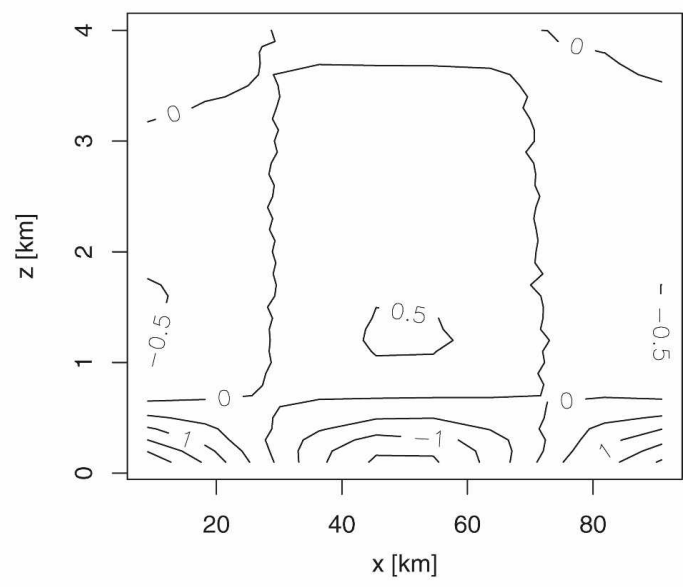

u(analytical); t= 6 [h];

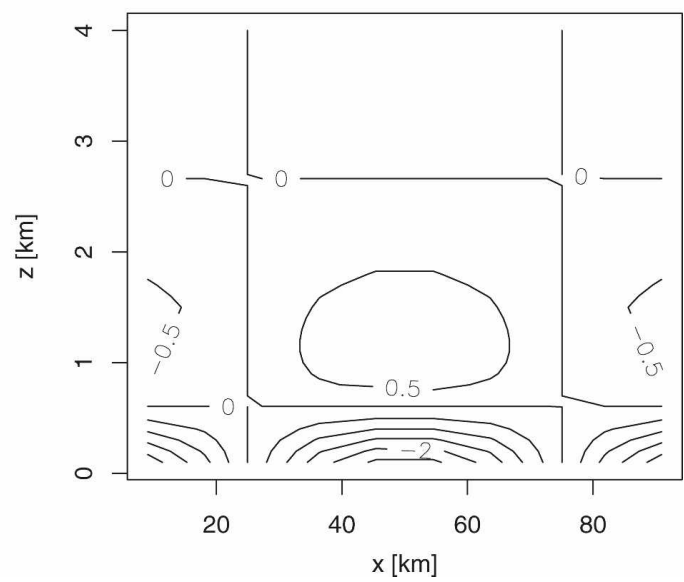

FIG. 3. Fully Lagrangian simulation of the sea-breeze system using stationary particles and neglecting the nonlinear advection terms. Numerical solutions are compared with Defant's analytical solution. (a) The potential temperature distribution $\theta(x, z, t=6 \mathrm{~h})$ and (b) the horizontal velocity field $u(x, z, t=6 \mathrm{~h})$. The labeled contour plot is used to show the agreement between the numerical results and the analytical results. A slight disagreement for both $u$ and $\theta$ near the model top is due to the numerical artifact of the rigid-lid boundary condition.

\section{c. Analysis of the nonlinear model}

To verify the proposed fully Lagrangian modeling approach, the above simulation is repeated for the seabreeze system so that the bracketed terms in Eqs. (8)(12) are fully exploited by advecting parcels with tagged velocity as described in section $2 b$.

Figure 4 presents contour plots of the potential temperature field $\theta(x, z, t=24 \mathrm{~h})$ and the horizontal velocity field $u(x, z, t=24 \mathrm{~h})$ for $M=1^{\circ} \mathrm{C}$ at $t=24 \mathrm{~h}$ (i.e., at the end of diurnal period of sea-breeze circulation). These plots confirm the absence of serious numerical artifacts in the Lagrangian simulation because simulated results are almost identical with their analytical analog. Good qualitative agreement between the top and bottom rows of Fig. 4 confirms the convergence of the nonlinear model simulations. This agreement can be understood as a consequence of setting $M=1^{\circ} \mathrm{C}$, which meant that the nonlinear terms in the numerical model were small, resulting in a quasi-linear prediction that is similar to the linear Defant solutions (Martin and Pielke 1983). Our choice of the small value of $M=$ $1{ }^{\circ} \mathrm{C}$ stems from our desire to ensure comparability between the analytical solutions and the Lagrangian model in the presence of nonlinear advection terms. Martin and Pielke (1983) reported using a nonlinear Eulerian model in which numerical velocity $u$ disagreed strongly with Defant's solution for larger values of $M$. For smaller values, say $M=1^{\circ} \mathrm{C}$, the results of the nonlinear Eulerian model agrees sufficiently with the analytical results (e.g., chapters 5 or 11 in Pielke 2002).

We now investigate how the Lagrangian model is 
(a)

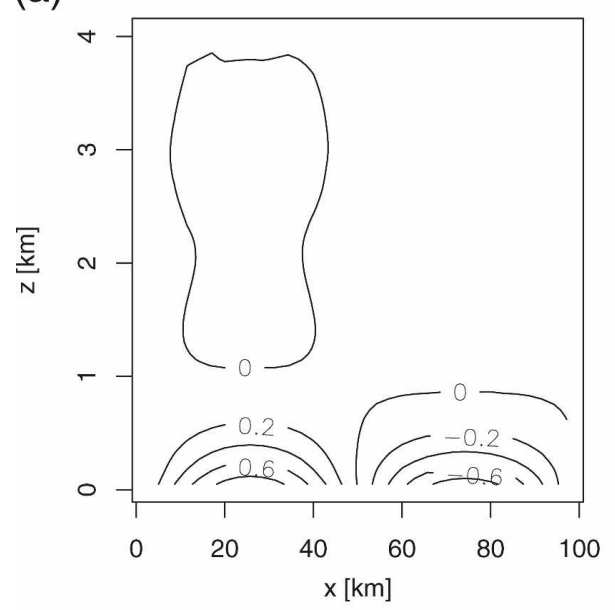

theta'(analytical); $\mathrm{t}=24$ [h];

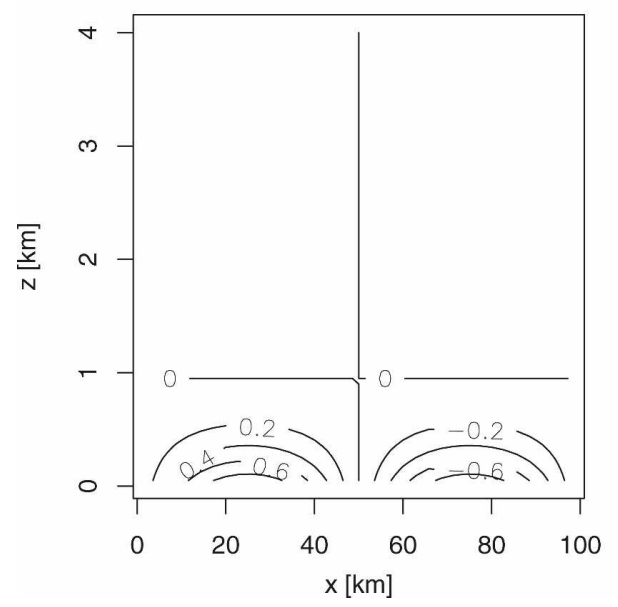

(b) u'(numerical); $\mathbf{t = 2 4}[\mathrm{h}]$;

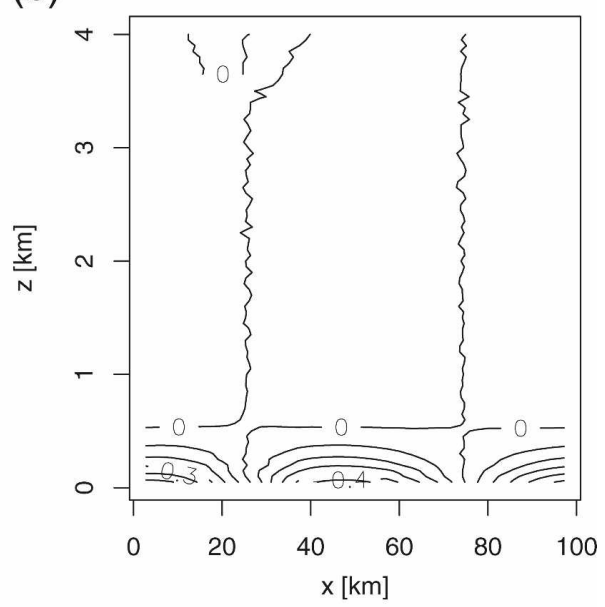

$u^{\prime}($ analytical); $\mathbf{t =} 24$ [h];

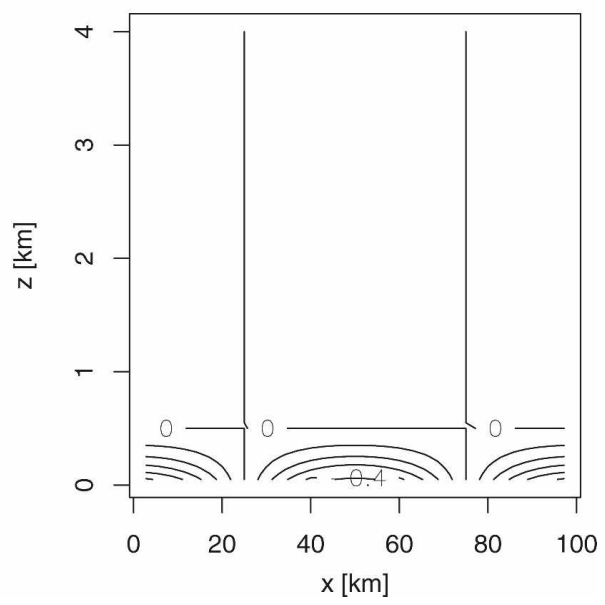

FIG. 4. Fully Lagrangian simulation of the sea-breeze system using nonstationary particles and including nonlinear advection terms. (a) The potential temperature distribution $\theta(x, z, t=24 \mathrm{~h})$ and (b) the horizontal velocity field $u(x, z, t=24 \mathrm{~h})$.

sensitive to the effect of the land-water temperature difference $M$. The sea-breeze intensity in coastal regions is observed to increase with the maximum landwater temperature difference (e.g., Defant 1951). This increases the nonlinear interaction between solution modes. In Fig. 5, we present the horizontal component of the velocity field for $M=1^{\circ}$ and $M=10^{\circ}$ calculated at the time of maximum heating which is about $6 \mathrm{~h}$ after sunrise. In this experiment, the horizontal scale of heating was $100 \mathrm{~km}$ and the vertical extent of the model was $10 \mathrm{~km}$. The larger vertical extent compared to previous experiments is used to see the region that is mostly affected by larger values of $M$. In each case, the nonlinear result is compared with its linear analog. We see good qualitative agreement between the nonlinear solution and the linear solution for $M=1^{\circ} \mathrm{C}$, but there is a strong disagreement for $M=10^{\circ} \mathrm{C}$. Because of the high velocity gradients around $x=25 \mathrm{~km}$ near the ground, strong mixing of air parcels occur for $M=$ $10^{\circ} \mathrm{C}$. Such mixing in the flow is not resolved without advection terms (e.g., chapter 11 in Pielke 2002). In contrast, structures in the linear solution are identical for both values of $M$ except for their magnitudes. Lagrangian simulation results are fully consistent with such physical characteristics of the sea-breeze circulation.

\section{d. Stability and convergence of the nonlinear model}

According to the Lax equivalence theorem, a consistent discretization of a well-posed linear initial value problem is convergent if and only if it is stable (chapter 3 in Richtmyer and Morton 1967). For nonlinear problems, the stability and convergence of a scheme can be verified if a small perturbation of the initial data does 
(a)

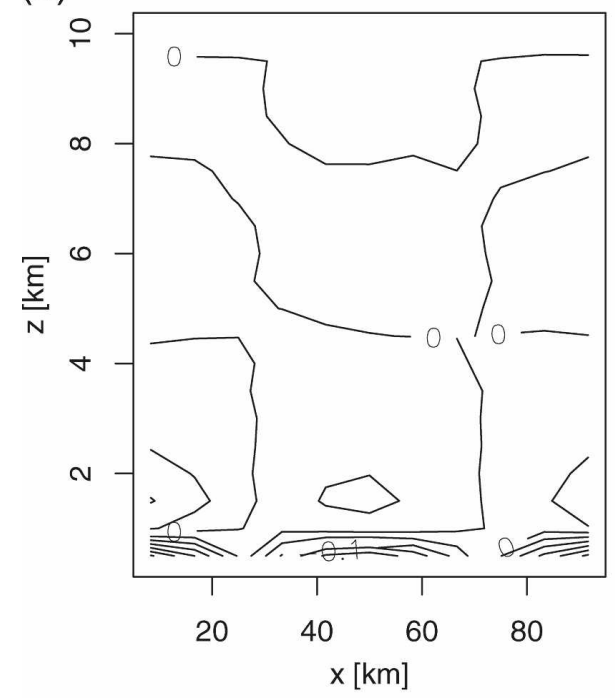

(b)

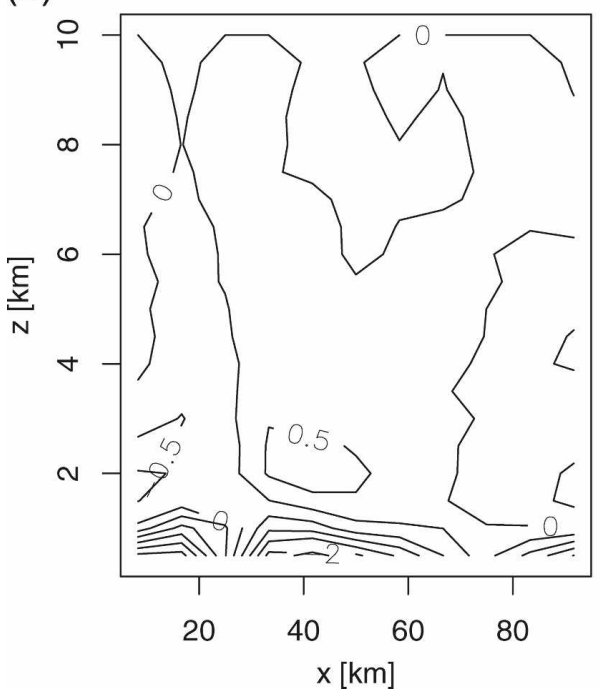

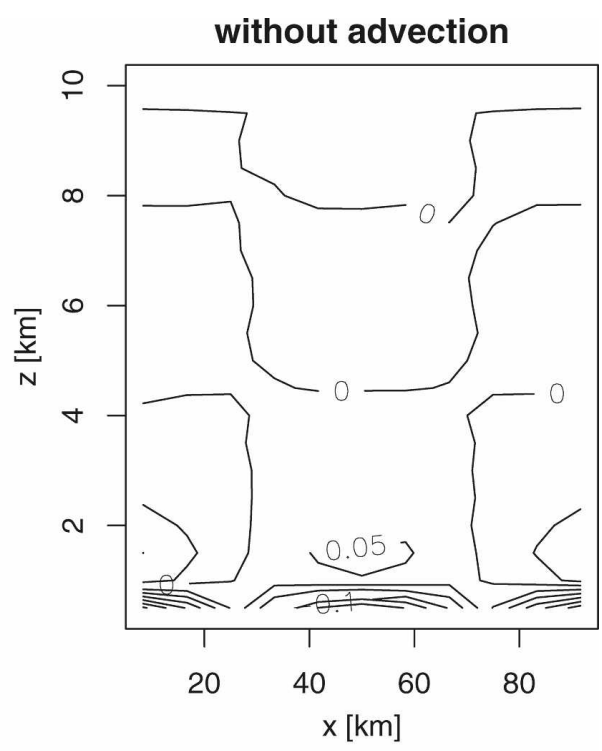

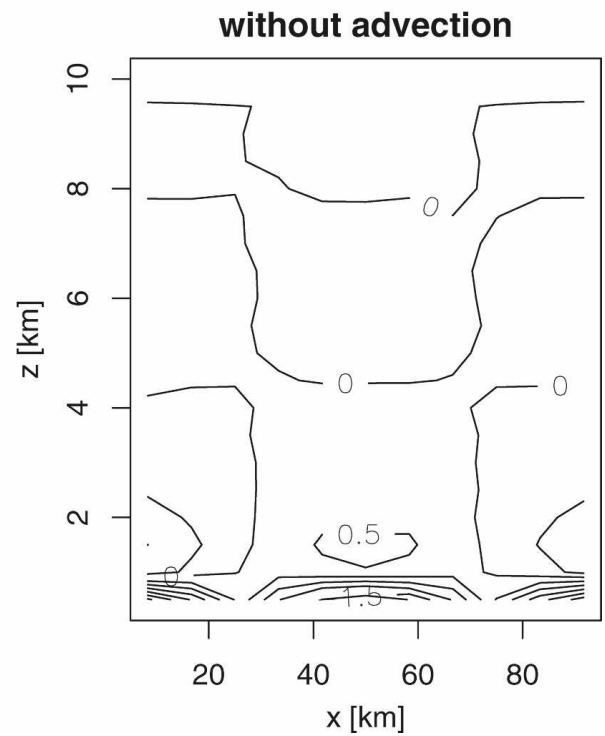

FIG. 5. The horizontal velocity field $6 \mathrm{~h}$ after sunrise with a maximum land-water temperature difference of (a) $M=1{ }^{\circ} \mathrm{C}$ and (b) $M=10^{\circ} \mathrm{C}$. Without nonlinear advection terms, the magnitude of the velocity field is proportional to $M$, but keeps similar structures. Including nonlinear advection terms causes both the structure and the magnitude of the velocity field to be changed.

not change the solution drastically in later times. In the present model, perturbation of the number of particles introduces perturbation to the numerical solution. Therefore, if the numerical model is unstable, a change in the particle number would introduce a relatively large change in the solution. The Lagrangian approach has thus one arbitrary aspect that requires further testing and understanding: the number of parcels to simulate in each grid cell.

A series of experiments are designed on a fixed staggered grid system by changing the average number $n$ of parcels that is assigned initially per grid cell. Figure 6 presents $w$ - the vertical component of the velocity field for $n=10,20,30,40,50$, and 60. For smaller values of $n$, fluctuations in the contour levels are evident, which are then eliminated as $n$ increases. However, the largescale characteristic of the solution is very similar for all values of $n$, because the same Eulerian grid is used to postprocess the data. Note that the total number of parcels used to calculate a flow is the total number of computational elements (i.e., the number of computational degrees of freedom). Using a fixed staggered grid system, if the average number $n$ of parcels per grid cell is small, fluctuations in the solution are expected, which 

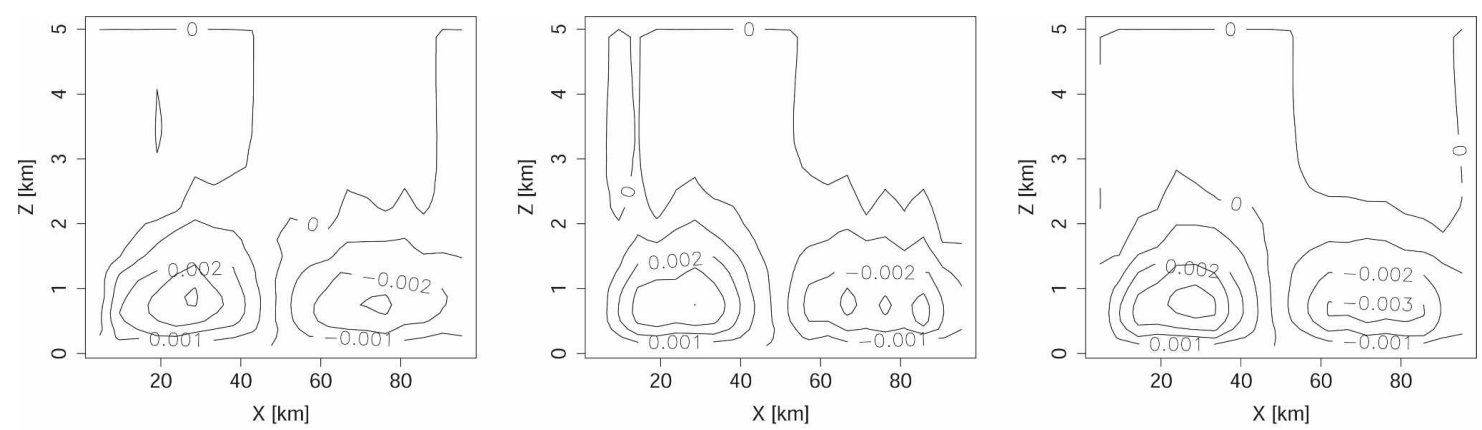

$n=10$

$n=20$
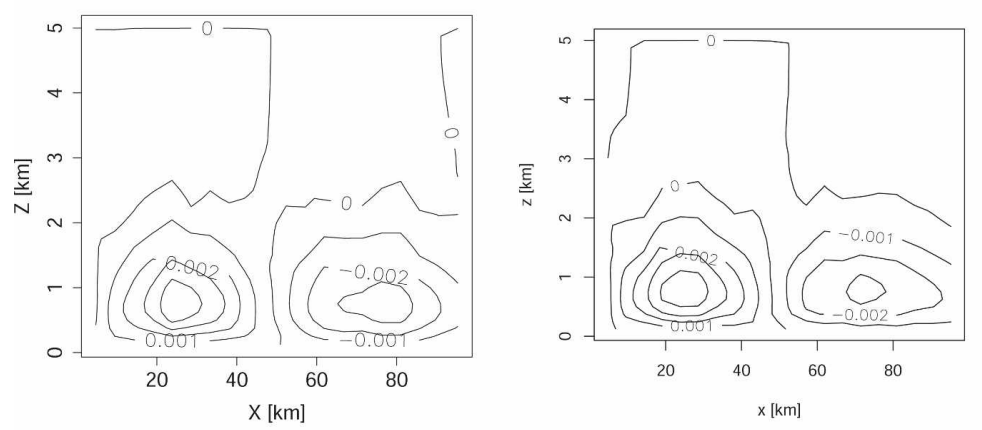

$n=40$

$n=50$

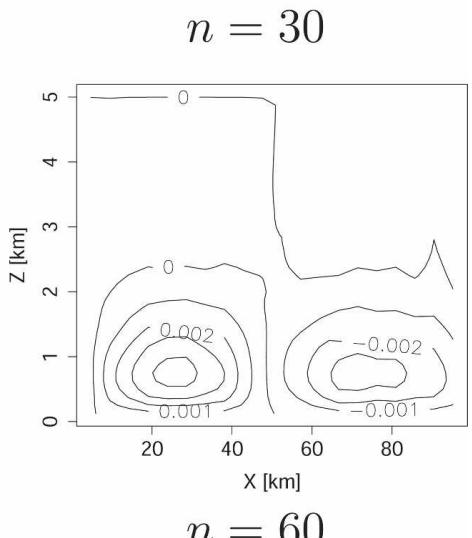

FIG. 6. Stability and convergence analysis of the Lagrangian model using a nonlinear sea-breeze circulation system. The vertical velocity component $w$ is computed by changing $n$, the number of parcels initially assigned at each grid cell. Clearly, fluctuations are present for smaller values of $n$, which are averaged out as $n$ increases.

tend to average out (see Fig. 6) quite effectively if the number of parcels increases (Harlow 1964).

To measure the accuracy, we compute the normalized root-mean-square error (rmse)

$$
\mathcal{E}_{\varphi}=\sqrt{\frac{\sum_{i}^{\mathcal{N}}\left(\varphi_{\mathrm{sim}, i}-\varphi_{\mathrm{ana}, i}\right)^{2} / \sum_{i}^{\mathcal{N}}\left(\varphi_{\mathrm{ana}, i}\right)^{2}}{\mathcal{N}}}
$$

of a dependent variable $\varphi$, where the subscripts "sim" and "ana" refer to "simulated" and "analytical" values, respectively; and $\mathcal{N}$ is the total number of grid points. Numerical simulations are conducted with $n=$ $10,20, \ldots, 100$ using a fixed $11 \times 20$ staggered grid system. The error of the sea-breeze simulation is calculated for each $n$ with respect to Defant's analytical solution.

Plots of rmse with varying $n$ are presented in Fig. 7 . The error is calculated with $M=1{ }^{\circ} \mathrm{C}$ and $M=10^{\circ} \mathrm{C}$ and comparing results from removing and retaining nonlinear terms in the sea-breeze system. Notice that the error is larger for smaller values of $n$, with a relatively sharp drop at near $n=20$, and the error decreases slowly for $n>20$ except for $M=10^{\circ} \mathrm{C}$ in the nonlinear case. Since the magnitude of an exact solution to Defant's linear model is proportional to $M$, but possesses the same functional form with respect to $x$ and $z$ for all values of $M$, the relative rmse is expected to be almost identical for both values of $M$ as depicted in Fig. 7. In contrast, when nonlinear terms are included into the sea-breeze system (Fig. 5), the spatial functional form of a solution must be affected if $M$ varies. Consequently, the maximum rmse of the nonlinear case for $M=10^{\circ} \mathrm{C}$ is expected to be larger compared to other cases. This is because we compare the Lagrangian model's simulation with Defant's analytical solution as a compromise to derive a rough measure of the accuracy since we do not have an exact solution for the nonlinear sea-breeze system.

One also expects that the error should be relatively small for simulations without advection, as confirmed from Fig. 7. Clearly, a rapid decrease of error is not associated with increasing $n$. There are a few reasons. First, the principal role of particles in a Lagrangian model is to provide more accurate solution in a future time such that the global accumulation of time integration error due to nonlinear advection is reduced by a significant factor using minimal computational cost, which is further verified and presented in section 4 . 


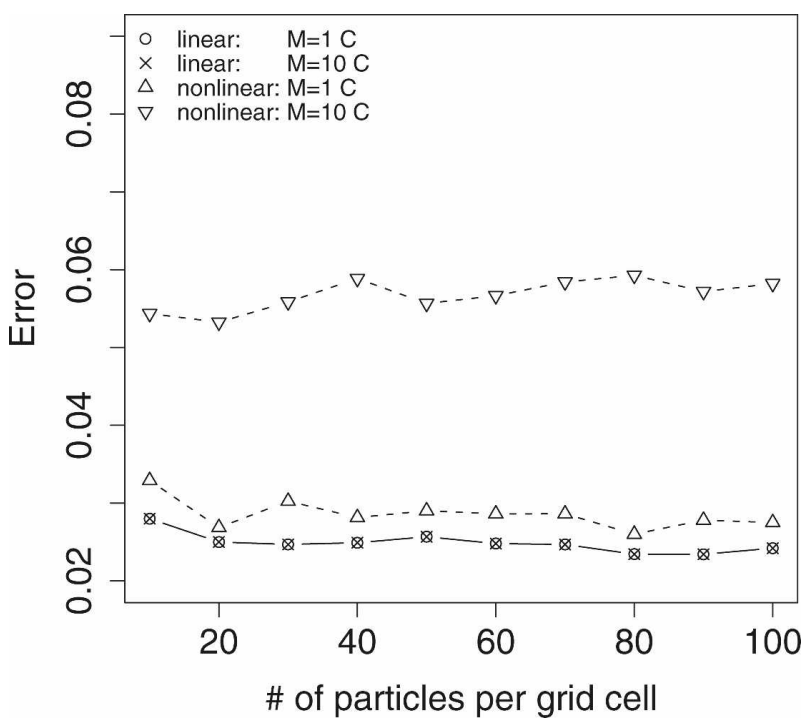

FIG. 7. The rmse $\varepsilon_{u}$ as a function of the number of particles for the Lagrangian model is computed with respect to the solution of Defant's sea-breeze model for $M=1{ }^{\circ} \mathrm{C}$ and $M=10^{\circ} \mathrm{C}$. Solutions of the linear Defant's model are compared with its nonlinear ana$\log$ presented in this paper. The normalized rmse is almost identical for both values of $M$ for the linear case, which is consistent with Defant's analytical solutions.

Second, the pressure gradient and diffusion operator for the potential temperature field are approximated at the grid points of the staggered grid in Fig. 2. Since the grid size $(\Delta x$ or $\Delta z)$ is larger than the smallest distance between nearby particles, the dominant error in these simulations is associated with the grid-based calculation of differential operators, and this can be reduced only by decreasing grid sizes, rather than increasing the particle number per grid cell. Third, the time evolution of all variables is calculated at subgrid-scale parcel position but the solution is projected back on to the grid points, thereby retaining the order of accuracy as a function of grid size ( $\Delta x$ and $\Delta z$ ). It is, however, important to examine the stability of the model in the sense that perturbations incurred by changing $n$ are not amplified, as demonstrated in Fig. 7. Clearly, the error follows roughly the same bound with varying $n$ for both values of $M$, which means that the nonlinear solution is convergent but does not converge to Defant's solution.

We note here an additional important finding. According to Fig. 7, a reasonable simulation using the proposed Lagrangian modeling system would require about 20 parcels per grid cell. These estimates show that about $\mathcal{N}=4400$ parcels are enough to simulate sea-breeze circulation in a coastal region using a twodimensional model with a $11 \times 20$ staggered grid system, but $\mathcal{N}$ would increase with a higher-resolution grid. The fully Lagrangian model's performance is fur- ther verified by comparing with an Eulerian and a semiLagrangian model.

\section{Comparison to Eulerian and semi-Lagrangian methods}

\section{a. Eulerian and semi-Lagrangian experiments}

We compare the developed fully Lagrangian model to semi-Lagrangian and Eulerian techniques for modeling advection in order to examine potential advantages and drawbacks. The semi-Lagrangian model uses Eq. (5) to calculate the trajectory of a fluid parcel that passes through each grid point at the present time. Clearly, the upstream position or "foot" of a trajectory is usually situated between grid points, requiring an interpolation of the solution from surrounding grid points (e.g., Staniforth and Cote 1991). The present semi-Lagrangian model uses a cubic spline interpolation technique (e.g., chapter 10 in Pielke 2002) to interpolate a solution at an upstream location. The Eulerian model is a finite-difference method presented in Harlow and Welch (1965) for two-dimensional incompressible flow calculation, where nonlinear terms are discretized in the flux form. Note that errors for both the semi-Lagrangian and Eulerian models are reduced by a factor of 2 if step sizes $(\Delta t$ or $\Delta x$ ) are refined by a factor of 2. The same is also true for the proposed fully Lagrangian model. The Eulerian model could be improved, for example, using a flux correction scheme, and similarly for the semi-Lagrangian model by using a higher-order scheme for trajectory calculation (e.g., chapter 5 in Durran 1998). Many researchers realized that more sophisticated schemes resulted in improvements of the approximation at significant expenses, where correcting one error would magnify another error (e.g., chapter 10 in Pielke 2002). The present work examines what differences could be made if a fully Lagrangian approach were taken instead of Eulerian or semi-Lagrangian approaches.

A series of numerical simulations for all three numerical methods have been carried out at the following grid configurations (horizontal $\times$ vertical): $5 \times 5,5 \times$ $10,10 \times 20,20 \times 40$, and $30 \times 60$. The model domain extends $100 \mathrm{~km}$ in east-west direction, covering $50 \mathrm{~km}$ over land, $50 \mathrm{~km}$ over water, and $4 \mathrm{~km}$ in the vertical direction. The horizontal and vertical grid spaces are $3.3 \mathrm{~km}$ and $133 \mathrm{~m}$, respectively, for the finest grid; and $20 \mathrm{~km}$ and $800 \mathrm{~m}$, respectively, for the coarsest grid. For the fully Lagrangian model, 30 particles were initially assigned at each grid cell. All state variables were equal to 0 at $t=0$, where the potential temperature was initialized according to (13) with $M=1^{\circ} \mathrm{C}$. Comparisons between the fully Lagrangian, semi-Lagrangian, 

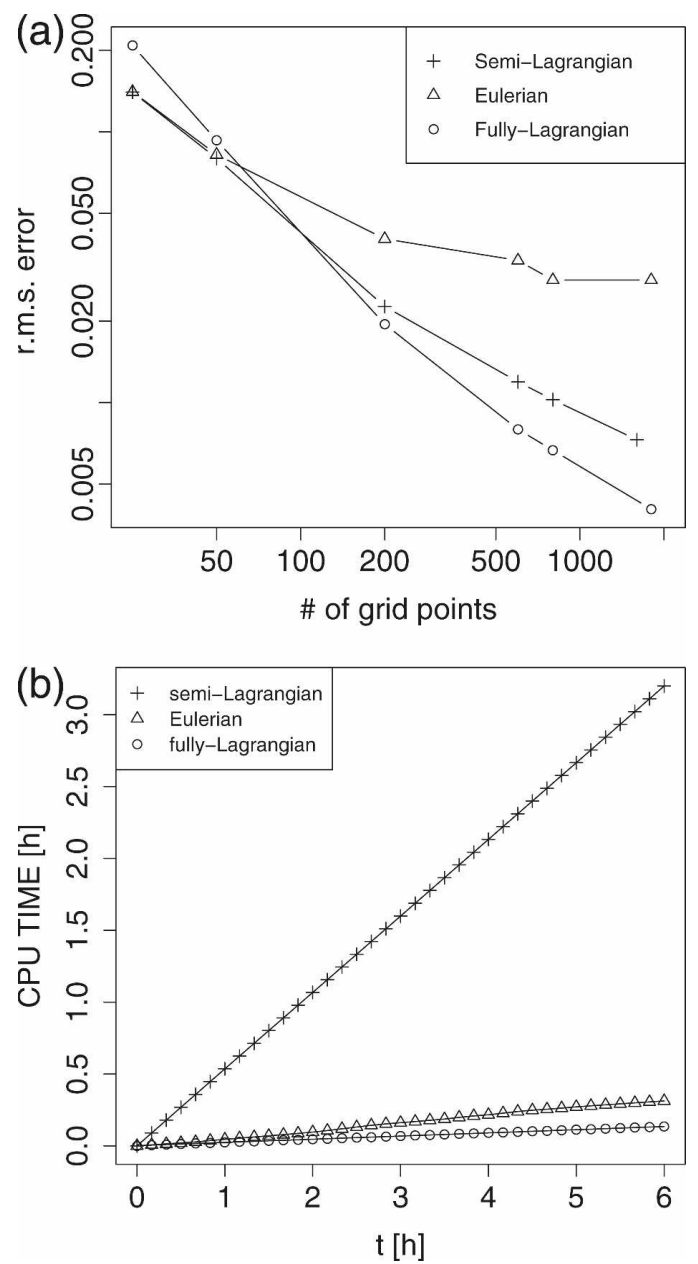

FIG. 8. (a) The rmse is compared for the semi-Lagrangian, Eulerian, and fully Lagrangian simulation (with 40 parcels initially assigned at each grid cell) of the sea-breeze system. The grid is refined successively starting from a coarse grid and the error as a function of the total number of grid points is calculated at each grid. (b) CPU time is compared among the semi-Lagrangian, Eulerian, and fully Lagrangian simulation of the sea-breeze system. Elapsed processor time (h) is recorded at each time step to show how much CPU time is used for any fixed simulation time $t$.

and Eulerian approaches were carried out in two aspects: 1) accuracy and 2) computational cost.

\section{b. Comparison for accuracy}

The normalized rmse [Eq. (14)] after $6 \mathrm{~h}$ of the seabreeze simulation was calculated and compared for fully Lagrangian, Eulerian, and semi-Lagrangian models. The dependence of the error on varying grid resolution is presented in Fig. $8 \mathrm{a}$ as a function of the number of grid points. For coarser grids, the resolution was not sufficient to resolve the flow. For increasing resolution, the reduction of error is roughly linear for both fully and semi-Lagrangian models. However, the error of the fully Lagrangian model remains smaller than that of the semi-Lagrangian model, and the difference is magnified with increasing resolution.

The larger errors for the Eulerian model on finer grids arise from numerical artifacts-diffusion and dispersion error associated with the finite-difference approximation. When the spatial grid is refined, stability conditions require that the time step must also be refined, increasing the required number of time steps. Given a time step $\Delta t$ that is proportional to the fastest time scale, local numerical artifacts can add up significantly such that the numerical solution becomes highly erroneous after a number of time steps. A balance between maximizing a time step and reducing such globally accumulated error leads to further reduction of a time step that was determined by stability restrictions. The present work determines a time step for the Eulerian model empirically based on some numerical experiments, where the time step for the Eulerian model was smaller by about a factor of 3 than the average time step used for the fully and semi-Lagrangian models. The accumulation of such numerical artifact is a principal drawback for conventional Eulerian schemes, and a better understanding of the rate at which such error accumulates with increasing number of time steps remains an active area of research as reported by many researchers in other areas dealing similar computational problems (e.g., see Knoll et al. 2006). Therefore, the error behavior of the Eulerian model is consistent with observations obtained by other researchers.

\section{c. Comparison for computational cost}

The elapsed CPU time (h) is recorded at a fixed time interval to examine how much processor time is required with respect to the wall clock. In Fig. 8b, a comparison of the recorded CPU time as a function of time elapsed in the model (with sunrise in the sea-breeze simulation denoted by $t=0$ ) is presented, which shows that the elapsed CPU time grows at a constant rate for all three models. Rates of increase of elapsed CPU time for the fully Lagrangian, Eulerian, and semiLagrangian models are $0.02,0.04$, and 0.53 , respectively, at the unit of CPU (h) per model time (h; slopes of three lines in Fig. 8b). This shows that the fully Lagrangian model is roughly 2 times as fast as the Eulerian model and is about 20 times as fast as the semiLagrangian model.

The higher computational cost of the Eulerian model versus that of the fully Lagrangian model stems from two sources. As stated in the previous section, the Eulerian model's time step is smaller by a factor of 3 . Second, the computation of the nonlinear terms on an 
Eulerian staggered grid increases the central processing unit (CPU) time (Harlow and Welch 1965). The higher cost for the semi-Lagrangian method is associated with cubic spline interpolation, which becomes more expensive for multidimensional cases (e.g., see chapter 10 in Pielke 2002). Note higher-order splines are standard for semi-Lagrangian interpolation although they incur considerable computational cost (e.g., Staniforth and Cote 1991; Ritchie 1986). For example, Bartello and Thomas (1996) reported that a semi-Lagrangian scheme usually trades about 5-10 times of computational work off to improve in accuracy by a significant factor compared to Eulerian schemes, as confirmed in this study. Results presented in Fig. 8 demonstrate that a fully Lagrangian model can employ a Lagrangian mesh of particles for accurate modeling of nonlinear advection process without significantly increasing the computational cost, suggesting a better alternative to conventional semiLagrangian approaches. This result would further encourage atmospheric scientists to develop improved atmospheric modeling systems based on the fully Lagrangian frame work.

\section{Summary and future developments}

\section{a. Summary}

This paper has investigated, for the first time, the possibility for a fully Lagrangian treatment of coupled equation of atmospheric dynamics in the Lagrangian frame at mesoscale-simulating both the velocity and advecting scalar fields simultaneously using a Lagrangian mesh of particles.

The performance of the developed fully Lagrangian model is compared with a semi-Lagrangian model and an Eulerian finite-difference model. These comparisons lead to the following important observations: (i) fully Lagrangian simulations are cost effective and accurate compared to the traditional semi-Lagrangian and Eulerian simulations; (ii) refining the grid provides greater error reduction for the fully Lagrangian model; and (iii) numerical behavior over long time integrations is much better for the fully Lagrangian approach with higherresolution grids - a clear step toward the development of efficient algorithms for large-scale computational problems.

\section{b. Future developments}

The findings in this paper point to future research directions. The success of convergence and stability verifications using a two-dimensional sea-breeze circulation model encourages extension of the developed Lagrangian method for three-dimensional simulations. Three-dimensional mixing processes such as Kelvin-
Helmholtz instability and frontal waves play an important role in momentum transfer or trace gas fluxes over the land surface (Garner 1989), but are missing from the current two-dimensional formulation. It would be necessary to resolve a wide range of length and time scales for the Lagrangian model to simulate threedimensional mixing processes, thereby incurring a steep computational cost. The cost for a semi-Lagrangian or an Eulerian model would also increase relatively for such simulation. Thus, one expects the fully Lagrangian model to be highly effective even for three-dimensional simulation, but this has yet to be verified numerically.

Recent work has demonstrated that the computational cost associated with resolving turbulent flows at high Reynolds number can be drastically reduced using a wavelet method that dynamically adapts the solution in both space and time (e.g., Kevlahan et al. 2007). In addition to grid adaptation, a wavelet method can improve accuracy with a fixed number of computational degrees of freedom by increasing the vanishing moments of wavelets. Particularly, a Lagrangian method can be guided to adopt an optimal mapping to grid points or the spatial distribution of particles by combining with an appropriate wavelet approximation (e.g., Bergdorf and Koumoutsakos 2006). However, this approach has yet to be implemented and verified in the context of atmospheric simulation.

The steep computational cost that may arise from large-scale two- or three-dimensional simulations can be handled using a suitable parallelization algorithm (e.g., Roberti et al. 2004) and/or adopting a simultaneous space-time adaptive procedure (Alam et al. 2006). The Lagrangian modeling approach is attractive for parallelization because calculations along fluid trajectories are independent of each other and can be easily distributed among processors.

Several additional steps are necessary to enhance the realism of the fully Lagrangian model. Water and turbulence need to be added to the current model. The model can also be extended to include tracer transport and chemical reactions between various tracers. This would enable the model to investigate important problems in air quality and atmospheric chemistry.

We have already undertaken efforts to incorporate a stochastic parameterization of turbulence and will soon test ways to add moisture and implement adaptive gridding. Ultimately, we envision a fully Lagrangian comprehensive atmospheric modeling system that can provide fundamental improvements in atmospheric simulations.

Acknowledgments. JCL thanks Daniel McKenna for first inspiring the possibility that the atmosphere can be 
simulated in an entirely Lagrangian manner. We acknowledge Nicholas Kevlahan, Yu-heng Tseng, and Roger Pielke Sr. for their insights and helpful comments on drafts of this paper. Author JA was partly supported by a grant from the Natural Sciences and Engineering Research Council of Canada. This work was made possible by the facilities of the Shared Hierarchical Academic Research Computing Network (SHAR-CNET: www.sharcnet.ca).

\section{REFERENCES}

Alam, J. M., 2000: A fully Lagrangian advection scheme for electro-osmotic flow. M.S. thesis, Dept. of Mathematical Sciences, University of Alberta, Alberta, Canada, $124 \mathrm{pp}$.

— , N. K.-R. Kevlahan, and O. Vasilyev, 2006: Simultaneous space-time adaptive solution of nonlinear parabolic differential equations. J. Comput. Phys., 214, 829-857.

Baptista, A., 1987: Solution of advection-dominated transport by Eulerian-Lagrangian methods using the backwards method of characteristics. Ph.D. thesis, Dept. of Civil Engineering, Massachusetts Institute of Technology, Cambridge, MA, 260 pp.

Bartello, P., and S. Thomas, 1996: The cost-effectiveness of semiLagrangian advection. Mon. Wea. Rev., 124, 2883-2897.

Bates, J. R., and A. McDonald, 1985: Comments on "Some properties and comparitive performance of the semi-Lagrangian method of Robert in the solution of the advection-diffusion equation." Atmos.-Ocean, 23, 193-194.

Behrens, J., 2006: Adaptive Atmospheric Modeling: Key Techniques in Grid Generation, Data Structures, and Numerical Operations with Applications. Springer, 214 pp.

Bergdorf, M., and P. Koumoutsakos, 2006: A Lagrangian particlewavelet method. Multiscale Model. Simulat., 5 (3), 980-995, doi: $10.1137 / 060652877$.

Bermejo, R., 1990: On the equivalence of semi-Lagrangian schemes and particle-in-cell finite element methods. Mon. Wea. Rev., 118, 979-987.

Chung, T. J., 2002: Computational Fluid Dynamics. Cambridge University Press, 1036 pp.

Cottet, G.-H., and P. D. Koumoutsakos, 2000: Vortex Methods: Theory and Practice. Cambridge University Press, 313 pp.

Crowley, W. P., 1968: Numerical advection experiments. Mon. Wea. Rev., 96, 1-11.

Defant, F., 1951: Local winds. Compendium of Meteorology, T. F. Malone, Ed., Amer. Meteor. Soc., 655-672.

Denning, A. S., G. J. Collatz, C. Zhang, D. A. Randall, J. A. Berry, P. J. Sellers, G. D. Collello, and D. A. Dazlich, 1996: Simulations of terrestrial carbon metabolism and atmospheric $\mathrm{CO}_{2}$ in a general circulation model. Part-I: Surface carbon fluxes. Tellus, 48B, 521-542.

Draxler, R. R., and G. D. Hess, 1998: An overview of the HYPSPLIT4 modeling system for trajectories, dispersion, and deposition. Aust. Meteor. Mag., 47, 295-308.

Dritschel, D. G., L. M. Polvani, and A. R. Mohebalhojeh, 1999: The contour-advective semi-Lagrangian algorithm for the shallow water equations. Mon. Wea. Rev., 127, 1551-1565.

Durran, D. R., 1998: Numerical Methods for Wave Equations in Geophysical Fluid Dynamics. Springer-Verlag, 465 pp.

Fast, J. D., and R. C. Easter, 2006: A Lagrangian particle disper- sion model compatible with WRF. Extended Abstracts, Seventh Annual WRF User's Workshop, Boulder, CO.

Garner, S. T., 1989: Fully Lagrangian numerical solutions of unbalanced frontogenesis and frontal colapse. J. Atmos. Sci., 46, 717-739.

Hack, J. J., 1992: Climate system simulations: Basic numerical and computational concepts. Climate System Modeling, K. Trenberth, Ed., Cambridge University Press, 283-318.

Haertel, P., and D. Randall, 2002: Could a pile of slippery sacks behave like an ocean. Mon. Wea. Rev., 130, 2975-2988.

$\longrightarrow,-$, and T. Jensen, 2004: Simulating upwelling in a large lake using slippery sacks. Mon. Wea. Rev., 132, 66-77.

Harlow, F. H., 1964: The Particle-in-Cell computing method for fluid dynamics. Methods Comput. Phys., 3, 319-343.

_- , and J. E. Welch, 1965: Numerical calculation of timedependent viscous incompressible flow of fluid with free surface. Phys. Fluids, 8 (12), 2182-2189.

Jacobson, M. Z., 1999: Fundamentals of Atmospheric Modeling. Cambridge University Press, 672 pp.

Jaouen, S., 2007: A purely Lagrangian method for computing linearly-perturbed flows in spherical geometry. J. Comput. Phys., 225, 464-490.

Kevlahan, N.-R., J. Alam, and O. Vasilyev, 2007: Scaling of spacetime modes with the Reynolds number in two-dimensional turbulence. J. Fluid Mech., 570, 217-226.

Knoll, D. A., V. A. Mousseau, R. M. Rauenzahn, and K. J. Evans, 2006: Multiphysics time integration and long time integration error. Tech. Rep. LA-UR-06-3716, Computational Sciences and Fluid Dynamics, Theoretical Division of Nuclear Weapons Program Highlights, Los Alamos National Laboratory, $81 \mathrm{pp}$.

Koumoutsakos, P., 2005: Multiscale flow simulations using particles. Annu. Rev. Fluid Mech., 37, 457-487, doi:10.1146/ annurev.fluid.37.061903.175753.

Krishnamurti, T. N., 1962: Numerical integration of primitive equations by a quasi-Lagrangian advection scheme. J. Appl. Meteor., 1, 508-521.

Kundu, P. K., 1990: Fluid Mechanics. Academic Press, 83 pp.

Lange, R., 1978: ADPIC-A three-dimensional particle-in-cell model for the dispersal of atmospheric pollutants and its comparison to regional tracer studies. J. Appl. Meteor., 17, 320-329.

Leonard, A., 1985: Computing three-dimensional incompressible flows with vortex elements. Annu. Rev. Fluid Mech., 17, 523559.

Lin, J. C., C. Gerbig, S. C. Wofsy, A. E. Andrews, B. C. Daube, K. J. Davis, and C. A. Grainger, 2003: A near-field tool for simulating the upstream influence of atmospheric observations: The Stochastic Time-Inverted Lagrangian Transport (STILT) model. J. Geophys. Res., 108, 4493, doi:10.1029/ 2002JD003161.

Lucy, L. B., 1977: A numerical approach to the testing of the Fisson Hypothesis. Astro. J., 82, 1013-1024.

Mahrer, Y., and R. A. Pielke, 1978: A test of an upstream spline interpolation technique for the advective terms in a numerical mesoscale model. Mon. Wea. Rev., 106, 818-830.

Martin, C. L., and R. A. Pielke, 1983: The adequacy of the hydrostatic assumption in sea breeze modeling over flat terrain. $J$. Atmos. Sci., 40, 1472-1481.

Mathur, M. B., 1983: A quasi-Lagrangian regional model designed for operational weather prediction. Mon. Wea. Rev., 111, 2087-2098. 
Monaghan, J., 1988: An introduction to SPH. Comput. Phys. Commun., 48, 89-96.

Oliveira, A., and A. M. Baptista, 1995: A comparison of integration and interpolation Eulerian-Lagrangian methods. Int. J. Numer. Methods Fluids, 21, 183-204.

Phillips, N. A., 1956: The general circulation of the atmosphere: A numerical experiment. Quart. J. Roy. Meteor. Soc., 82, 123164.

Pielke, R. A., 2002: Mesoscale Meteorological Modeling. Academic Press, 676 pp.

Pudykiewicz, J., and A. Staniforth, 1984: Some properties and comparative performance of the semi-Lagrangian method of Robert in the solution of the advection-diffusion equation. Atmos.-Ocean, 22, 283-308.

Read, P. L., N. P. J. Thomas, and S. H. Risch, 2000: An evaluation of Eulerian and semi-Lagrangian advection schemes in simulations of rotating, stratified flows in the laboratory. Part I: Axisymmetric flow. Mon. Wea. Rev., 128, 2835-2852.

Richtmyer, R. D., and K. W. Morton, 1967: Difference Methods for Initial-Value Problems. Interscience Publishers, 401 pp.

Ritchie, H., 1986: Eliminating the interpolation associated with the semi-Lagrangian scheme. Mon. Wea. Rev., 114, 135-146.

Roberti, D. R., R. P. Souto, H. F. de Campos Velho, G. A. Degrazia, and D. Anfossi, 2004: Parallel implementation of a Lagrangian stochastic model for pollution dispersion. $S B A C$ PAD '04: Proc. 16th Symp. on Computer Architecture and High Performance Computing (SBAC-PAD'04), Washington, DC, IEEE Computer Society, 142-149. [Available online at http://ieeexplore.ieee.org/search/wrapper.jsp?arnumber $=$ 1364747.]

Rood, R. B., 1987: Numerical advection algorithms and their role in atmospheric transport and chemistry models. Rev. Geophys., 25, 71-100.

Ryall, D., and R. Maryon, 1998: Validation of the UK Met. Office's NAME model against the ETEX dataset. Atmos. Environ., 32, 4265-4276.

Sawford, B. L., 1985: Lagrangian statistical simulation of concentration mean and fluctuation fields. J. Climate Appl. Meteor., 24, 1152-1166.

Sawyer, J. S., 1963: A semi-Lagrangian method of solving the vorticity advection equation. Tellus, 15, 336-342.
Shen, J., 1992: On error estimates of some higher order projection and penalty-projection methods for Navier-Stokes equations. Numer. Math., 62, 49-73.

Sherman, C. A., 1978: A mass-consistent model for wind fields over complex terrain. J. Appl. Meteor., 17, 312-319.

Smolarkiewicz, P. K., and J. A. Pudykiewicz, 1992: A class of semi-Lagrangian approximations for fluids. J. Atmos. Sci., 49, 2082-2096.

Staniforth, A., and J. Pudykiewicz, 1985: Reply to comments on and addenda to "Some properties and comparative performance of the semi-Lagrangian method of Robert in the solution of the advection-diffusion equation." Atmos.-Ocean, 23, 195-200.

_- and J. Cote, 1991: Semi-Lagrangian integration schemes for atmospheric models-A review. Mon. Wea. Rev., 119, 22062223.

Stohl, A., and D. J. Thomson, 1999: A density correction for Lagrangian particle dispersion models. Bound.-Layer Meteor., 90, 155-167.

_- M. Hittenberger, and G. Wotawa, 1998: Validation of the Lagrangian particle dispersion model FLEXPART against large-scale tracer experiment data. Atmos. Environ., 32, 4245-4264.

Subramaniam, S., 1996: A new mesh-free vortex method. Ph.D. thesis, Florida State University, 239 pp.

Tannehill, J. C., D. A. Anderson, and R. H. Pletcher, 1997: Computational Fluid Mechanics and Heat Transfer. 2d ed. Taylor and Francis, 816 pp.

Uliasz, M., 1996: Regional modeling of air pollution transport in the southwestern United States. Environmental Modelling: Computer Methods and Software for Simulating Environmental Pollution and Its Adverse Effects, P. Zannetti, Ed., Computational Mechanics, 145-181.

Wang, Y., and K. Hutter, 2001: Comparisons of numerical methods with respect to convectively dominated problems. Int. J. Numer. Methods Fluids, 37 (6), 721-745.

Wiin-Nielsen, A., 1959: On the application of trajectory methods in numerical forecasting. Tellus, 11, 180-196.

Wilson, J. D., and B. L. Sawford, 1995: Review of Lagrangian stochastic models for trajectories in the turbulent atmosphere. Bound.-Layer Meteor., 78, 191-210. 